\title{
Demonstration of a Large Dissipative Haptic Environment
}

\author{
Mike Vande Weghe ${ }^{1}$, Brian Dellon ${ }^{2}$, Sean Kelly ${ }^{2}$, Richard Juchniewicz ${ }^{2}$, Yoky Matsuoka ${ }^{1,2}$ \\ ${ }^{1}$ Robotics Institute, ${ }^{2}$ Mechanical Engineering \\ Carnegie Mellon University \\ 5000 Forbes Ave, Pittsburgh, PA 15213 \\ email: \{michaelv,btd,sjkelly,rjuchnie,yoky\}@andrew.cmu.edu
}

\begin{abstract}
Herein we describe a hands-on demonstration presented at the 2006 Symposium on Haptic Interfaces. Our device is a dissipative six degree-offreedom haptic robot with a workspace of 2 cubic meters. By using dissipative actuators we are able to increase user safety. Our demonstration allows subjects to evaluate many of the haptic features that are required for a rich haptic environment.
\end{abstract}

\section{Introduction}

General-purpose haptic feedback devices are frequently used for assistance during manual procedures (virtual fixtures in surgery), product design (virtual clay, tactile evaluation), technique training, and rehabilitation. Because of concerns for user safety, most devices limit the power of their energetic actuators (electric motors, hydraulic or pneumatic cylinders) and thus are restricted to low-mass structures and small workspaces. Although largeworkspace devices have been built and used (such as MIME [1], WAM [2], PHANTOM [3], and HapticMaster [4]), these machines either carry the risk of user injury caused by a malfunction, or limit their speed, force and/or range-of-motion to levels insufficient for some applications (such as rehabilitation). Our research interest is in exploring the performance capabilities of a device that utilizes passive actuators for motion control. Specifically for our first step, we constructed a six degrees-of-freedom (DOF) haptic environment similar to work previously done by one of the current authors [5]. The device has a workspace of approximately 2 cubic meters, and utilizes dissipative brake actuators for motion control in order to safeguard against user injury. We show through demonstration that even when the device is purely dissipative, many features desired in a generalpurpose haptic environment can be realized with orthogonal kinematics, axes alignment and knowledge of human perception and motor control.

\section{Mechanical Design}

Previous work $[5,6]$ has shown that robots using only non-energetic actuators must have orthogonal kinematics in order to control all the forces in the Cartesian space of the user. Our design (figure 1) implements a six DOF device. The three braked DOF restrict the positioning of the endpoint based on a spherical coordinate system $(r, \theta, \phi)$, while three free DOF built into the handle assembly allow for roll, pitch, and yaw rotations of the user's hand with respect to the endpoint. The device is intended to accommodate most movements made with a subject's hand and arm while keeping their torso stationary, and has a $\mathrm{min} / \mathrm{max}$ extension $(r)$ of $0.25 / 1.0 \mathrm{~m}$, with theta and phi travel of $+/-60^{\circ}$ (total workspace of approx. 2 cubic meters).

The device is designed to resist a maximum of $134 \mathrm{~N}$ of force at the endpoint while at full extension $(r=1.0 \mathrm{~m})$, equivalent to a torque of $134 \mathrm{Nm}$.

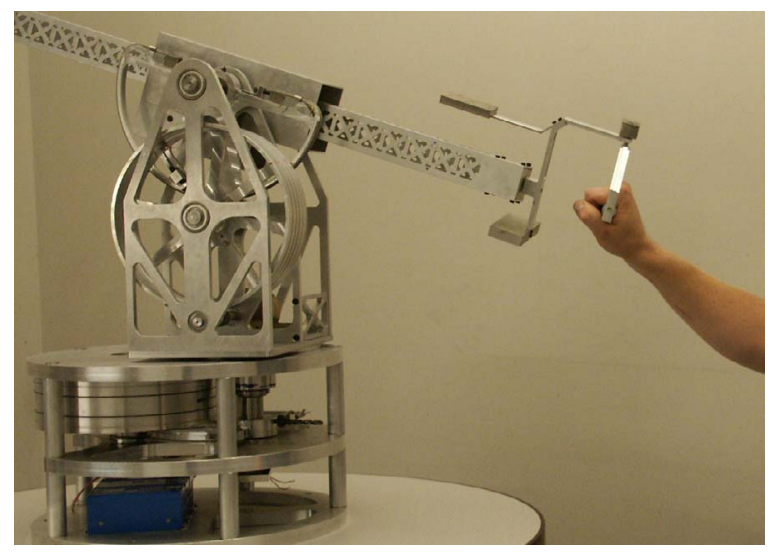

Figure 1: The 6-DOF dissipative haptic device. 
Instead of using massive particle brakes rated for the full torque load, the design uses smaller, lighter brakes in conjunction with low-mass cable reduction drives. The two-stage cable drives on the theta and phi axes provide an 82:1 reduction in torque, yielding a resultant max torque on the brakes of only $1.63 \mathrm{Nm}$. The reduction in the turret mass substantially reduces the rotational inertia about the vertical axis, creating a device which feels much lighter and more responsive. Because the $r$-axis of the machine does not have an associated large moment arm, the $134 \mathrm{~N}$ max force can be resisted with only a simple linear-to-rotational translation stage.

Our three DOF handle was designed to eliminate singularities by using a non-orthogonal gimbal design (figure 2) in which no two axes could align with one another. The yaw axis of the handle is rotated $20^{\circ}$ with respect to the roll axis, creating a conical dead zone about the roll axis. Each stage of the handle uses counterweights calculated to keep the assembly dynamically balanced about each of the DOF so that rotations do not produce any off-axis reaction forces. The resulting design provides for pitch rotation of $+150^{\circ} \% 180^{\circ}$, yaw of $+135^{\circ} \% 180^{\circ}$, and roll of $360^{\circ}$.

Because activation of the prismatic stage shifts its center-of-gravity with respect to the pitch axis, we have implemented a variable gravity compensation system to maintain zero net force on the user. The system uses a tensioned spring and cable similar to [7] with the addition of a small DC motor that adjusts the cable endpoint to maintain balance as the prismatic stage shifts. Since the motor moves the endpoint almost perpendicular to the cable, only a small fraction of the motor energy can be conveyed to the user, and user safety is still maintained.

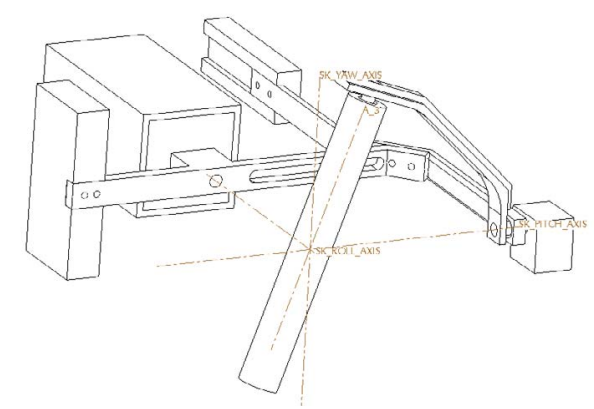

Figure 2. 3-DOF handle design with its rotated yaw axis to prevent gimbal lock.

\section{Sensing and Control}

The device uses optical encoders on each of the three actuated axes, and a six-axis force/torque sensor at the coupling between the device arm and the handle assembly. During development an additional singleaxis torque sensor was employed in series with each of the particle brakes in order to calibrate endpoint forces with braking forces. The magnetic particle brakes are activated via linear power amplifiers. Low-level control is achieved by calculating the position, velocity, and acceleration of the endpoint, comparing the values to a map of obstacles, force fields, and dampers, and adjusting the braking levels accordingly.

\section{Performance}

Our orthogonal kinematics allow for a smooth continuous workspace where the resisted forces can be varied freely. The demonstration depicts virtual geometric objects with both "sticky" and "non-sticky" surfaces [5] in a gravity-free environment.

\section{Acknowledgement}

This work has been funded by NSF grant 0238204 .

\section{References}

[1] P. S. Lum, C. G. Burgar, H. F. M. Van der Loos, "The use of a robotic device for post-stroke movement therapy," Proceedings of the International Conference on Rehabilitation Robotics, April 14-15, 1997. Bath, U.K.

[2] WAM robot, Barrett Technology, Inc, http://www.barretttechnology.com

[3] PHANTOM Premium 3.0 Haptic Device, Sensable Technologies, Inc, http://www.sensable.com

[4] R. Q. Van der Linde, P. Lammertse., E. Frederisksen, and B. Ruiter, "The HapticMaster, a new high-performance haptic interface," Proc. Eurohaptics 2002, pp. 1-5, July 8-10, Edinburgh, UK

[5] Y. Matsuoka and W. T. Townsend, "Design of lifesize haptic environments", Experimental Robotics VII, Rus and Singh, ed, 2001.

[6] R. B. Gillespie, J. E. Colgate, M. Peshkin, "A General Framework for Cobot Control," Proc. 1999 International Conference on Robotics and Automation, pp. 1824-1830.

[7] T. Rahman, R. Ramanathan, R. Seliktar, and W. Harwin, "A Simple Technique to Passively Gravity-Balance Articulated Mechanisms," ASME Transactions on Mechanisms Design, Vol 117(4), pp 655-658, December 1995. 